\title{
On the order of the reduction of a point on an abelian variety
}

\section{Journal Article}

Author(s):

Pink, Richard

Publication date:

2004-10

Permanent link:

https://doi.org/10.3929/ethz-b-000160332

Rights / license:

In Copyright - Non-Commercial Use Permitted

Originally published in:

Mathematische Annalen 330(2), https://doi.org/10.1007/s00208-004-0548-8 


\title{
On the order of the reduction of a point on an abelian variety
}

\section{Richard Pink}

Received: 30 November 2003 / Published online: 23 June 2004 - (c) Springer-Verlag 2004

\begin{abstract}
Consider a point of infinite order on an abelian variety over a number field. Then its reduction at any place $v$ of good reduction is a torsion point. For most of this paper we fix a rational prime $\ell$ and study how the $\ell$-part of this reduction varies with $v$. Under suitable conditions we prove various statements on this $\ell$-part for all $v$ in a set of positive Dirichlet density: for example that its order is a fixed power of $\ell$, that its order is non-trivial for the reductions of finitely many points, or that its order is larger than a certain explicit value that varies with $v$. By similar methods we prove that for all $v$ in a set of positive Dirichlet density the reduction of a given abelian variety possesses no non-trivial supersingular abelian subvariety.
\end{abstract}

Mathematics Subject Classification (2000): 14K15 (11R45)

\section{Introduction}

Consider an abelian variety $A$ over a number field $K$ and a rational point of infinite order $a \in A(K)$. Then the reduction $a_{v}$ of $a$ at any place $v$ of good reduction is defined over the finite residue field $k_{v}$ and is therefore a torsion point. It is natural to ask how $a_{v}$ varies with $v$. For most of this paper we fix a rational prime $\ell$ and study the $\ell$-part of $a_{v}$. Since for $v \nmid \ell$ any $\ell$-power torsion point over $\bar{k}_{v}$ possesses a unique $\ell$-power torsion lift to $A(\bar{K})$, one can try to translate this question into one over $\bar{K}$. The main player in this game is the group

$$
\ell^{-\infty}(\mathbb{Z} a):=\left\{x \in A(\bar{K}) \mid \exists n \geq 0: \ell^{n} x \in \mathbb{Z} a\right\} .
$$

This group is a natural extension of $\mathbb{Z}[1 / \ell]$ with the group of $\ell$-power torsion points

$$
A\left[\ell^{\infty}\right]:=\left\{x \in A(\bar{K}) \mid \exists n \geq 0: \ell^{n} x=0\right\} .
$$

The latter group has been studied extensively by means of the Galois representation on the associated $\ell$-adic Tate module $T_{\ell}(A)$. The former group also gives rise to a Tate module $T_{\ell}(A, a)$ which is an extension of $T_{\ell}(A)$ by $\mathbb{Z}_{\ell}$. It is a special case of the Tate modules of 1-motives introduced by Deligne [7, §10.1].

R. PINK

Department of Mathematics, ETH-Zentrum, CH-8092 Zürich, Switzerland (e-mail: pink@math.ethz.ch) 
Let $\Gamma_{\ell} \subset \operatorname{Aut}_{\mathbb{Z}_{\ell}}\left(T_{\ell}(A)\right)$ and $\tilde{\Gamma}_{\ell} \subset \operatorname{Aut}_{\mathbb{Z}_{\ell}}\left(T_{\ell}(A, a)\right)$ be the respective images of $\operatorname{Gal}(\bar{K} / K)$.

In Section 1 we review some known general facts about $\Gamma_{\ell}$ and its Zariski closure. We also prove in Corollary 1.7 that for all $v$ in a set of positive Dirichlet density the reduction of $A$ possesses no non-trivial supersingular abelian subvariety. Although this statement has no direct relation with the results on $a_{v}$, the respective methods of proof have much in common.

General structural properties of $\tilde{\Gamma}_{\ell}$ are then discussed in Section 2. In particular we recall Theorem 2.8 from the Kummer theory of $A$ which states that $\tilde{\Gamma}_{\ell}$ is an extension of $\Gamma_{\ell}$ by an open subgroup of $T_{\ell}(B)$, where $B$ is the identity component of the Zariski closure of $\mathbb{Z} a$. This result is essentially due to Ribet [13], though in the case we need the proof was worked out only by Hindry [9, §2, Prop. 1].

In Section 3 we then show how the $\ell$-part of $a_{v}$ is determined by the action of the Frobenius element $\operatorname{Frob}_{v}$ on $\ell^{-\infty}(\mathbb{Z} a)$. Any question about this $\ell$-part can thus be translated completely into a question on the group $\tilde{\Gamma}_{\ell}$.

In Section 4 we answer some of these questions. In all cases we prove that a certain behavior occurs for all places $v$ of $K$ in a set of Dirichlet density $>0$. For example, in Corollary 4.3 we show that under mild conditions every power of $\ell$ occurs as the order of the $\ell$-part of $a_{v}$. In Theorem 4.4 we prove that for finitely many given points $a_{i}$ of infinite order, the $\ell$-parts of their reductions $a_{i, v}$ can be made simultaneously non-trivial on a set of positive Dirichlet density. Theorem 4.7 generalizes this result in another direction: Let $f(T) \in \mathbb{Z}[T]$ be any polynomial which is a product of cyclotomic polynomials and a power of $T$. Let $p_{v}$ denote the residue characteristic at $v$. Then for suitable $\ell$, the $\ell$-parts of all $f\left(p_{v}\right) a_{i, v}$ can be made simultaneously non-trivial on a set of positive Dirichlet density.

In the final section 5 we use these theorems to derive two density results on the $a_{i, v}$ which no longer refer to any particular prime $\ell$. These results as well as Corollary 1.7 are needed in joint work with Damian Roessler [12] and provided the motivation for the present paper. ${ }^{1}$ Theorem 5.1 can also be deduced from work by Wong [16] who, instead of studying when the $\ell$-part of $a_{v}$ is zero, considers the dual question of when $a_{v}$ lies in $\ell \cdot A_{v}\left(k_{v}\right)$. Related questions are addressed in work by Corrales-Rodrigáñez and Schoof [6], Khare and Prasad [10], and Larsen [11].

\section{The $\ell$-adic Galois group associated to an abelian variety}

Let $K$ be a number field and $\bar{K}$ an algebraic closure of $K$. Consider an abelian variety $A$ of dimension $g$ over $K$ and a rational prime $\ell$. Then

$$
A\left[\ell^{\infty}\right]:=\left\{x \in A(\bar{K}) \mid \exists n \geq 0: \ell^{n} x=0\right\}
$$

1 The author wishes to thank Damian Roessler for the very fruitful ongoing collaboration. 
is a discrete group isomorphic to $\left(\mathbb{Q}_{\ell} / \mathbb{Z}_{\ell}\right)^{2 g}$ with a continuous action of $\operatorname{Gal}(\bar{K} / K)$. One usually describes this action via the $\ell$-adic Tate module

$$
T_{\ell}(A):=\operatorname{Hom}\left(\mathbb{Q}_{\ell} / \mathbb{Z}_{\ell}, A\left[\ell^{\infty}\right]\right) \cong \mathbb{Z}_{\ell}^{2 g},
$$

which possesses a continuous Galois representation

$$
\rho_{\ell}: \operatorname{Gal}(\bar{K} / K) \longrightarrow \operatorname{Aut}_{\mathbb{Z}_{\ell}}\left(T_{\ell}(A)\right) \cong \mathrm{GL}_{2 g}\left(\mathbb{Z}_{\ell}\right) .
$$

We are interested in its image $\Gamma_{\ell}:=\rho_{\ell}(\operatorname{Gal}(\bar{K} / K))$, which is a compact subgroup of $\mathrm{GL}_{2 g}\left(\mathbb{Z}_{\ell}\right)$. Much can be said about $\Gamma_{\ell}$ by means of its Zariski closure $G_{\ell} \subset \mathrm{GL}_{2 g, \mathbb{Q}_{\ell}}$. This is a linear algebraic group over $\mathbb{Q}_{\ell}$ with a natural faithful representation on the rational Tate module

$$
V_{\ell}(A):=T_{\ell}(A) \otimes_{\mathbb{Z}_{\ell}} \mathbb{Q}_{\ell} \cong \mathbb{Q}_{\ell}^{2 g} .
$$

The following general facts are known about $G_{\ell}$.

Theorem 1.1. (a) The action of $G_{\ell}$ on $V_{\ell}(A)$ is semisimple and the natural homomorphism

$$
\operatorname{End}_{K}(A) \otimes_{\mathbb{Z}} \mathbb{Q}_{\ell} \longrightarrow \operatorname{End}_{\mathbb{Q}_{\ell}, G_{\ell}}\left(V_{\ell}(A)\right)
$$

is an isomorphism.

(b) $G_{\ell}$ is a reductive group.

(c) $\Gamma_{\ell}$ is an open subgroup of $G_{\ell}\left(\mathbb{Q}_{\ell}\right)$.

Proof. By the definition of $G_{\ell}$ the statements in (a) are equivalent to the corresponding ones with $\Gamma_{\ell}$ in place of $G_{\ell}$, which were proved by Faltings [8, Th. 3-4]. Part (b) follows from the first statement in (a). Part (c) is a theorem of Bogomolov [4], [3].

By Galois theory every open subgroup of $\Gamma_{\ell}$ corresponds to a finite extension of $K$ within $\bar{K}$, and replacing $K$ by that extension amounts to replacing $\Gamma_{\ell}$ by the corresponding subgroup. In particular, let $G_{\ell}^{\circ}$ denote the identity component of $G_{\ell}$. Then replacing $\Gamma_{\ell}$ by any open subgroup of $\Gamma_{\ell} \cap G_{\ell}^{\circ}$ has the effect of replacing $G_{\ell}$ by $G_{\ell}^{\circ}$; and thereafter $G_{\ell}$ will be connected.

Now consider any finite place $v$ of $K$ and let $p_{v}$ denote the characteristic of the finite residue field $k_{v}$. If $v \nmid \ell$ and $A$ has good reduction at $v$, it is known that the restriction of $\rho_{\ell}$ to any inertia group above $v$ is trivial. Let Frob ${ }_{v}$ be any element of a decomposition group at $v$ which acts by taking $\left|k_{v}\right|^{\text {th }}$ powers modulo $v$. Then the conjugacy class of $\rho_{\ell}\left(\operatorname{Frob}_{v}\right)$ depends only on $v$ and is known to be semisimple, and its characteristic polynomial on $V_{\ell}(A)$ is known to have coefficients in $\mathbb{Z}$ and to be independent of $\ell$.

Choose any semisimple element $t_{v} \in \mathrm{GL}_{2 g}(\mathbb{Q})$ whose characteristic polynomial is equal to that of $\rho_{\ell}\left(\operatorname{Frob}_{v}\right)$. Let $T_{v} \subset \mathrm{GL}_{2 g, \mathbb{Q}}$ be the Zariski closure of the subgroup generated by $t_{v}$. The construction implies that the identity component 
of $T_{v}$ is a torus and its $\mathrm{GL}_{2 g}(\mathbb{Q})$-conjugacy class depends only on $v$. Following Serre [14] it is called the Frobenius torus at $v$. Moreover, for any $\ell \neq p_{v}$ there is a unique conjugate of $T_{v, \mathbb{Q}_{\ell}}$ by an element of $\mathrm{GL}_{2 g}\left(\mathbb{Q}_{\ell}\right)$ which lies in $G_{\ell}$, such that $t_{v}$ is mapped to $\rho_{\ell}\left(\right.$ Frob $\left._{v}\right)$. Serre [14, §5, pp.12-13] proves:

Theorem 1.2. If $G_{\ell}$ is connected, then for all places $v$ in a set of Dirichlet density 1 the group $T_{v}$ itself is a torus and $T_{v, \mathbb{Q}_{\ell}}$ is conjugate under $\mathrm{GL}_{2 g}\left(\mathbb{Q}_{\ell}\right)$ to a maximal torus of $G_{\ell}$.

Corollary 1.3. There exists a set of rational primes $\ell$ of positive Dirichlet density for which $G_{\ell}$ splits over $\mathbb{Q}_{\ell}$.

Proof. Let $T_{v}$ be any Frobenius torus as in Theorem 1.2. Choose a finite extension $F$ of $\mathbb{Q}$ such that $T_{v, F}$ splits. Then the set of rational primes $\ell$ which split completely in $F$ has positive Dirichlet density, and for each of them $T_{v, \mathbb{Q}_{\ell}}$ splits. Since $T_{v, \mathbb{Q}_{\ell}}$ is conjugate to a maximal torus of $G_{\ell}$, this shows that $G_{\ell}$ splits.

Next any polarization of $A$ induces a Galois equivariant perfect alternating pairing $V_{\ell}(A) \times V_{\ell}(A) \rightarrow \mathbb{Q}_{\ell}(1)$, where $\operatorname{Gal}(\bar{K} / K)$ acts on $\mathbb{Q}_{\ell}(1)$ through the cyclotomic character. It follows that $\Gamma_{\ell}$ is contained in the group of symplectic similitudes $\mathrm{CSp}_{2 g}\left(\mathbb{Q}_{\ell}\right)$. Let $\mu: \mathrm{CSp}_{2 g} \rightarrow \mathbb{G}_{m}$ denote the multiplier map; then $\mu \rho_{\ell}: \operatorname{Gal}(\bar{K} / K) \rightarrow \mathbb{Z}_{\ell}^{*}$ is the cyclotomic character. The definition of $G_{\ell}$ implies that $G_{\ell} \subset \mathrm{CSp}_{2 g}, \mathbb{Q}_{\ell}$; hence $\mu$ defines an algebraic character of $G_{\ell}$.

Proposition 1.4. Consider a maximal torus $S_{\ell}$ of $G_{\ell}$ and any weight $\chi$ of $S_{\ell}$ on $V_{\ell}(A)$. Then $\mu$ and $\chi$ are $\mathbb{Q}$-linearly independent in the character group of $S_{\ell}$.

Proof. The perfect pairing implies that there exists a weight $\chi^{*}$ of $S_{\ell}$ on $V_{\ell}(A)$ such that $\chi \chi^{*}=\mu$. Both $\chi$ and $\chi^{*}$ are non-trivial, because the corresponding Frobenius eigenvalues have complex absolute value $>1$. Now by the Hodge-Tate decomposition there exists a cocharacter $\lambda$ of $S_{\ell}$ whose weights on $V_{\ell}(A)$ are 0 and 1 and whose weight on $\mathbb{Q}_{\ell}(1)$ is 1 ; see for instance Serre [14, §5, pp.11-12]. For any such $\lambda$ we have

$$
\langle\chi, \lambda\rangle+\left\langle\chi^{*}, \lambda\right\rangle=\left\langle\chi \chi^{*}, \lambda\right\rangle=\langle\mu, \lambda\rangle=1
$$

and one of the summands is 0 and the other 1 . This implies that $\chi$ and $\chi^{*}$ cannot be non-zero rational multiples of each other. Since they are both non-trivial characters, they must be $\mathbb{Q}$-linearly independent. Equivalently $\chi$ and $\mu=\chi \chi^{*}$ are $\mathbb{Q}$-linearly independent, as desired.

Proposition 1.5. Suppose that $A=A_{1} \times \ldots \times A_{d}$ for non-zero abelian varieties $A_{1}, \ldots, A_{d}$. Consider a maximal torus $S_{\ell}$ of $G_{\ell}$. Then there exist weights $\chi_{i}$ of $S_{\ell}$ on $V_{\ell}\left(A_{i}\right)$ so that $\mu$ is $\mathbb{Q}$-linearly independent of $\chi_{1}, \ldots, \chi_{d}$. 
Proof. By the Hodge-Tate decomposition, see [14, §5, pp.11-12], there exists a cocharacter $\lambda$ of $S_{\ell}$ which on every $V_{\ell}\left(A_{i}\right)$ has the weights 0 and 1 with multiplicity $\operatorname{dim} A_{i}$ each, and whose weight on $\mathbb{Q}_{\ell}(1)$ is 1 . So we can choose each $\chi_{i}$ such that $\left\langle\chi_{i}, \lambda\right\rangle$, the weight of the $\chi_{i}$-eigenspace in the Hodge-Tate decomposition, is zero. Then for any weight $\chi$ which is a $\mathbb{Q}$-linear combination of the $\chi_{i}$, we still have $\langle\chi, \lambda\rangle=0$. But $\langle\mu, \lambda\rangle=1$; hence $\mu$ is not a $\mathbb{Q}$-linear combination of the $\chi_{i}$.

We finish this section with a first application of Proposition 1.4, which will not be used in the rest of the paper.

Theorem 1.6. If $G_{\ell}$ is connected, the set offinite places $v$ of $K$ where the reduction of A does not possess a non-trivial supersingular abelian subvariety has Dirichlet density 1.

Proof. By Theorem 1.2 it suffices to consider those places $v \nmid \ell$ of $K$ for which $T_{v, \mathbb{Q}_{\ell}}$ is conjugate to a maximal torus $S_{\ell}$ of $G_{\ell}$. Let $v$ be such a place and suppose that the corresponding reduction $A_{v}$ of $A$ possesses a non-trivial supersingular abelian subvariety $B_{v}$. Then any eigenvalue of Frob ${ }_{v}$ on $V_{\ell}\left(B_{v}\right)$ has the form $\sqrt{\left|k_{v}\right|}$ times a root of unity, while the eigenvalue on $\mathbb{Q}_{\ell}(1)$ is $\left|k_{v}\right|$. Let $\chi$ be the weight of $S_{\ell}$ on $V_{\ell}(A)$ corresponding to that eigenvalue on $V_{\ell}\left(B_{v}\right) \subset V_{\ell}\left(A_{v}\right)$, and let $n$ be the order of that root of unity. Then the values of $\chi^{2 n}$ and $\mu^{n}$ on $\rho_{\ell}\left(\right.$ Frob $\left._{v}\right)$ coincide. But by the construction of the Frobenius torus the element $\rho_{\ell}\left(\right.$ Frob $\left._{v}\right)$ generates a Zariski dense subgroup of $S_{\ell}$. Thus $\chi^{2 n}$ and $\mu^{n}$ are equal as characters of $S_{\ell}$, which contradicts their linear independence from Proposition 1.4. This shows that $A_{v}$ does not possess a non-trivial supersingular abelian subvariety, as desired.

Corollary 1.7. Let $A$ be an abelian variety over a number field $K$. Then there exists a finite extension $L$ of $K$ such that for all finite places of $L$ in a set of Dirichlet density 1 the reduction of $A$ does not possess a non-trivial supersingular abelian subvariety.

Proof. Choose an arbitrary rational prime $\ell$ and a finite Galois extension $L$ of $K$ over which $G_{\ell}$ becomes connected, and apply Theorem 1.6.

\section{The $\ell$-adic Galois group associated to an abelian variety with a point}

Now fix a rational point of infinite order $a \in A(K)$ and set

$$
\ell^{-\infty}(\mathbb{Z} a):=\left\{x \in A(\bar{K}) \mid \exists n \geq 0: \ell^{n} x \in \mathbb{Z} a\right\} .
$$

Then we have a natural short exact sequence of discrete groups

$$
0 \longrightarrow A\left[\ell^{\infty}\right] \longrightarrow \ell^{-\infty}(\mathbb{Z} a) \stackrel{a \mapsto 1}{\longrightarrow} \mathbb{Z}[1 / \ell] \longrightarrow 0
$$


Any choice of a compatible system of $\ell$-power roots of $a$ determines a splitting $\lambda: \mathbb{Z}[1 / \ell] \rightarrow \ell^{-\infty}(\mathbb{Z} a)$ satisfying $\lambda(1)=a$. We will call such a splitting special. Two special splittings differ by an element of

$$
\operatorname{Hom}\left(\mathbb{Z}[1 / \ell] / \mathbb{Z}, A\left[\ell^{\infty}\right]\right) \cong \operatorname{Hom}\left(\mathbb{Q}_{\ell} / \mathbb{Z}_{\ell}, A\left[\ell^{\infty}\right]\right)=T_{\ell}(A)
$$

By contrast, two general splittings differ by an element of

$$
\begin{aligned}
\operatorname{Hom}\left(\mathbb{Z}[1 / \ell], A\left[\ell^{\infty}\right]\right) & =\bigcup_{r \geq 0} \operatorname{Hom}\left(\mathbb{Z}[1 / \ell] / \ell^{r} \mathbb{Z}, A\left[\ell^{\infty}\right]\right) \\
& \cong \bigcup_{r \geq 0} \ell^{-r} \operatorname{Hom}\left(\mathbb{Q}_{\ell} / \mathbb{Z}_{\ell}, A\left[\ell^{\infty}\right]\right) \\
& =\bigcup_{r \geq 0} \ell^{-r} T_{\ell}(A)=V_{\ell}(A) .
\end{aligned}
$$

The sequence 2.1 is equivariant under the natural continuous action of $\operatorname{Gal}(\bar{K} / K)$, where the action on $\mathbb{Z}[1 / \ell]$ is trivial. It is useful to describe this action via an associated Tate module. For this note that $\ell^{-\infty}(\mathbb{Z} a) / \mathbb{Z} a$ is isomorphic to $\left(\mathbb{Q}_{\ell} / \mathbb{Z}_{\ell}\right)^{2 g+1}$; hence

$$
T_{\ell}(A, a):=\operatorname{Hom}\left(\mathbb{Q}_{\ell} / \mathbb{Z}_{\ell}, \ell^{-\infty}(\mathbb{Z} a) / \mathbb{Z} a\right)
$$

is isomorphic to $\mathbb{Z}_{\ell}^{2 g+1}$ and sits in a short exact sequence

$$
0 \longrightarrow T_{\ell}(A) \longrightarrow T_{\ell}(A, a) \longrightarrow \mathbb{Z}_{\ell} \longrightarrow 0 .
$$

Any special splitting of 2.1 determines a splitting of 2.3, i.e., an isomorphism $T_{\ell}(A, a) \cong T_{\ell}(A) \oplus \mathbb{Z}_{\ell}$. We will write any such decomposition in terms of column vectors. Then the natural Galois representation on $T_{\ell}(A, a)$ has the form

$$
\tilde{\rho}_{\ell}=\left(\begin{array}{cc}
\rho_{\ell} & * \\
0 & 1
\end{array}\right): \operatorname{Gal}(\bar{K} / K) \longrightarrow\left(\begin{array}{cc}
\operatorname{Aut}_{\mathbb{Z}_{\ell}}\left(T_{\ell}(A)\right) & T_{\ell}(A) \\
0 & 1
\end{array}\right) \cong\left(\begin{array}{cc}
\mathrm{GL}_{2 g}\left(\mathbb{Z}_{\ell}\right) \mathbb{Z}_{\ell}^{2 g} \\
0 & 1
\end{array}\right) .
$$

The construction implies that left multiplication by the same matrices also describes the Galois action on $\ell^{-\infty}(\mathbb{Z} a) \cong A\left[\ell^{\infty}\right] \oplus \mathbb{Z}[1 / \ell]$. We are interested in the image

$$
\tilde{\Gamma}_{\ell}:=\tilde{\rho}_{\ell}(\operatorname{Gal}(\bar{K} / K)) \subset\left(\begin{array}{cc}
\Gamma_{\ell} & T_{\ell}(A) \\
0 & 1
\end{array}\right) .
$$

Letting $N_{\ell}:=\tilde{\Gamma}_{\ell} \cap T_{\ell}(A)$ denote its intersection with the upper right corner, we obtain a natural short exact sequence

$$
0 \longrightarrow N_{\ell} \longrightarrow \tilde{\Gamma}_{\ell} \longrightarrow \Gamma_{\ell} \longrightarrow 1 \text {. }
$$

As with $\Gamma_{\ell}$ we will study $\tilde{\Gamma}_{\ell}$ with the help of its Zariski closure $\tilde{G}_{\ell}$, which is a linear algebraic group over $\mathbb{Q}_{\ell}$ with a natural faithful representation on

$$
V_{\ell}(A, a):=T_{\ell}(A, a) \otimes_{\mathbb{Z}_{\ell}} \mathbb{Q}_{\ell} \cong \mathbb{Q}_{\ell}^{2 g+1} .
$$


By construction we have a natural short exact sequence

$$
0 \longrightarrow U_{\ell} \longrightarrow \tilde{G}_{\ell} \longrightarrow G_{\ell} \longrightarrow 1
$$

where $U_{\ell}$ is an algebraic subgroup of the vector group $V_{\ell}(A)$. Since $G_{\ell}$ is reductive by Theorem 1.1 (b), the subgroup $U_{\ell}$ is simply the unipotent radical of $\tilde{G}_{\ell}$.

Proposition 2.5. $\tilde{\Gamma}_{\ell}$ is open in $\tilde{G}_{\ell}\left(\mathbb{Q}_{\ell}\right)$ and $N_{\ell}$ open in $U_{\ell}\left(\mathbb{Q}_{\ell}\right)$.

Proof. By construction we have an inclusion of short exact sequences

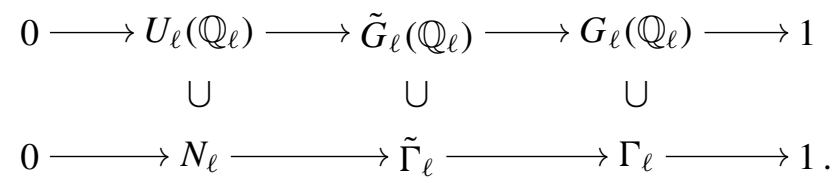

All these groups can be viewed as $\ell$-adic Lie groups, and by a theorem of Chevalley [5, Ch. II, Cor. 7.9] the Zariski density of $\tilde{\Gamma}_{\ell}$ implies

$$
\left[\operatorname{Lie} \tilde{G}_{\ell}, \text { Lie } \tilde{G}_{\ell}\right] \subset \operatorname{Lie} \tilde{\Gamma}_{\ell} .
$$

On the other hand $V_{\ell}(A)$ does not contain the trivial representation of $G_{\ell}$, because all Frobenius eigenvalues have complex absolute value $>1$. Thus $U_{\ell} \cong \operatorname{Lie} U_{\ell}$ does not contain the trivial representation of $G_{\ell}$, which implies that

$$
\text { Lie } U_{\ell}=\left[\operatorname{Lie} \tilde{G}_{\ell}, \operatorname{Lie} U_{\ell}\right] \subset\left[\operatorname{Lie} \tilde{G}_{\ell}, \operatorname{Lie} \tilde{G}_{\ell}\right] \subset \operatorname{Lie} \tilde{\Gamma}_{\ell} .
$$

Since moreover Lie $\Gamma_{\ell}=$ Lie $G_{\ell}$ by Theorem 1.1 (c), we deduce that Lie $\tilde{\Gamma}_{\ell}=$ Lie $\tilde{G}_{\ell}$. Thus $\tilde{\Gamma}_{\ell}$ is open in $\tilde{G}_{\ell}\left(\mathbb{Q}_{\ell}\right)$, and therefore $N_{\ell}$ is open in $U_{\ell}\left(\mathbb{Q}_{\ell}\right)$, as desired.

Proposition 2.6. After replacing $K$ by a suitable finite extension there exists a splitting of 2.1, not necessarily special, such that

$$
\tilde{\Gamma}_{\ell}=\left(\begin{array}{cc}
\Gamma_{\ell} & N_{\ell} \\
0 & 1
\end{array}\right) .
$$

Proof. Choose any Levi decomposition $\tilde{G}_{\ell}=G_{\ell} \ltimes U_{\ell}$ and consider the short exact sequence

$$
0 \longrightarrow V_{\ell}(A) \longrightarrow V_{\ell}(A, a) \longrightarrow \mathbb{Q}_{\ell} \longrightarrow 0
$$

deduced from 2.3 by tensoring with $\mathbb{Q}_{\ell}$. As $G_{\ell}$ is reductive, acts trivially on $\mathbb{Q}_{\ell}$, and non-trivially on every non-zero subspace of $V_{\ell}(A)$, the sequence 2.7 possesses a unique splitting that is invariant under the Levi subgroup $G_{\ell}$. On the other hand take any splitting $\lambda$ of 2.1. Then the induced splitting of 2.7 differs from the Levi invariant splitting by some element of $V_{\ell}(A)$. Changing $\lambda$ by the same element thus shows that the Levi invariant splitting of 2.7 comes from some splitting of 
2.1, though not necessarily from a special one. With respect to this splitting the decomposition $\tilde{G}_{\ell}=G_{\ell} \ltimes U_{\ell}$ is the same as that in terms of formal matrices

$$
\tilde{G}_{\ell}=\left(\begin{array}{cc}
G_{\ell} & U_{\ell} \\
0 & 1
\end{array}\right) .
$$

Finally Proposition 2.5 implies that

$$
\left(G_{\ell}\left(\mathbb{Q}_{\ell}\right) \cap \tilde{\Gamma}_{\ell}\right) \ltimes\left(U_{\ell}\left(\mathbb{Q}_{\ell}\right) \cap \tilde{\Gamma}_{\ell}\right)
$$

is an open subgroup of $\tilde{G}_{\ell}\left(\mathbb{Q}_{\ell}\right)$ and hence of $\tilde{\Gamma}_{\ell}$. After replacing $K$ by the corresponding finite extension $\tilde{\Gamma}_{\ell}$ itself is such a semidirect product, as desired.

Theorem 2.8. Let $B$ be the identity component of the Zariski closure of $\mathbb{Z} a$. Then $N_{\ell}$ is open in $T_{\ell}(B) \subset T_{\ell}(A)$ and we have $U_{\ell}=V_{\ell}(B) \subset V_{\ell}(A)$.

Proof. This is a special case of a theorem essentially due to Ribet [13] on the Kummer theory of $A$, itself depending on results of Faltings [8] and Serre [15] as well as the Mordell-Weil theorem, and following a method first used by Bashmakov [1]. The case we need was formulated by Bertrand [2, Th. 2] and worked out by Hindry [9, §2, Prop. 1].

We begin with two technical reductions required by this reference. First, as the Mordell-Weil group $A(K)$ is finitely generated, the given element $a$ is an integral multiple of an indivisible element $a^{\prime} \in A(K)$. Replacing $a$ by $a^{\prime}$ does not change $B$, and since $\ell^{-\infty}(\mathbb{Z} a) \subset \ell^{-\infty}\left(\mathbb{Z} a^{\prime}\right)$ is a subgroup of finite index prime to $\ell$, it also changes neither $\tilde{\Gamma}_{\ell}$ nor $N_{\ell}$ nor $U_{\ell}$. Thus without loss of generality we may, and do, assume that $a$ itself is indivisible in $A(K)$. Next let $d$ be the number of connected components of the Zariski closure of $\mathbb{Z} a$. To prove the theorem we may, and do, replace $K$ by its finite extension $K(A[d])$.

Now for any two integers $r \geq s \geq 0$ consider the finite quotients

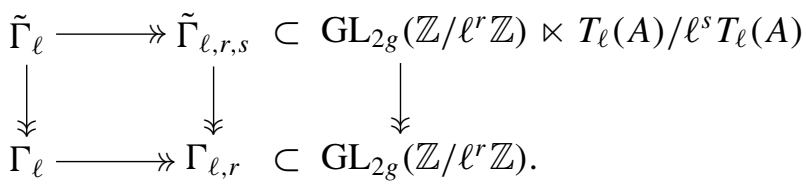

Then the short exact sequence 2.4 maps onto a short exact sequence

$$
0 \longrightarrow N_{\ell, r, s} \longrightarrow \tilde{\Gamma}_{\ell, r, s} \longrightarrow \Gamma_{\ell, r} \longrightarrow 1
$$

for some subgroup $N_{\ell, r, s} \subset T_{\ell}(A) / \ell^{s} T_{\ell}(A) \cong A\left[\ell^{s}\right]$. By [9, §2, Prop. 1] this group is a subgroup of $T_{\ell}(B) / \ell^{s} T_{\ell}(B) \cong B\left[\ell^{s}\right]$ whose index is bounded independently of $r$ and $s$, provided that $r \geq \operatorname{ord}_{\ell}(d)$. Since $N_{\ell}$ is the projective limit of the $N_{\ell, r, s}$ as both $r$ and $s$ go to infinity, this implies that $N_{\ell}$ is an open subgroup of $T_{\ell}(B)$. The second statement follows from this and Proposition 2.5. 
In particular, since $a$ has infinite order by assumption, Theorem 2.8 implies that $N_{\ell} \neq 0$. Another direct consequence is:

Corollary 2.9. $N_{\ell}$ is open in $T_{\ell}(A)$ if and only if $U_{\ell}=V_{\ell}(A)$ if and only if $\mathbb{Z} a$ is Zariski dense in A.

\section{The $\ell$-part of the reduction at $v$}

Now consider a place $v \nmid \ell$ of $K$ where $A$ has good reduction $A_{v}$. Then the restriction of $\tilde{\rho}_{\ell}$ to any inertia group above $v$ is trivial, and so the conjugacy class of $\rho_{\ell}\left(\operatorname{Frob}_{v}\right)$ depends only on $v$. We will show how this conjugacy class determines the $\ell$-part of the reduction $a_{v} \in A_{v}$ of our fixed point $a$.

First the condition $v \nmid \ell$ implies that the reduction map induces an isomorphism

$$
A\left[\ell^{\infty}\right] \stackrel{\sim}{\longrightarrow} A_{v}\left(\bar{k}_{v}\right)\left[\ell^{\infty}\right] .
$$

Consider the composite homomorphism

$$
\kappa_{v}: \ell^{-\infty}(\mathbb{Z} a) \subset A(\bar{K}) \longrightarrow A_{v}\left(\bar{k}_{v}\right) \longrightarrow A_{v}\left(\bar{k}_{v}\right)\left[\ell^{\infty}\right] \cong A\left[\ell^{\infty}\right],
$$

where the first arrow is reduction modulo $v$, the second one is the projection to the $\ell$-part, and the isomorphism on the right is the inverse of the reduction map. By construction its restriction to $A\left[\ell^{\infty}\right]$ is the identity, so $\kappa_{v}$ induces a splitting of the sequence 2.1. It is important to note that $\kappa_{v}$ does not in general correspond to a special splitting. Indeed, it does so if and only if $\kappa_{v}(a)=0$, that is, if the $\ell$-part of the reduction $a_{v}$ vanishes.

By construction $\kappa_{v}$ is equivariant under the action of Frob ${ }_{v}$. Thus the following observation tells us that $\kappa_{v}$ is completely determined by the element $\tilde{\rho}_{\ell}\left(\mathrm{Frob}_{v}\right) \in$ $\tilde{\Gamma}_{\ell}$.

Proposition 3.1. For every place $v \nmid \ell$ of $K$ where $A$ has good reduction the homomorphism $\kappa_{v}$ is the unique $\mathrm{Frob}_{v}$-equivariant splitting of the sequence 2.1.

Proof. Any other Frob $_{v}$-equivariant splitting $\ell^{-\infty}(\mathbb{Z} a) \rightarrow A\left[\ell^{\infty}\right]$ differs from

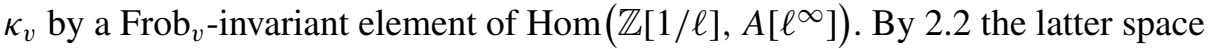
is isomorphic to $V_{\ell}(A)$. Since all eigenvalues of $\operatorname{Frob}_{v}$ on $V_{\ell}(A)$ have complex absolute value $>1$, its subspace of Frob ${ }_{v}$-invariants is zero. Thus $\kappa_{v}$ is the unique Frob $_{v}$-invariant splitting.

To give a precise formula for $\kappa_{v}(a)$ we fix a special splitting $\lambda$ of 2.1 and write

$$
\tilde{\gamma}_{v}:=\tilde{\rho}_{\ell}\left(\operatorname{Frob}_{v}\right)=\left(\begin{array}{cc}
\gamma_{v} & n_{v} \\
0 & 1
\end{array}\right)
$$

with $\gamma_{v}=\rho_{\ell}\left(\right.$ Frob $\left._{v}\right) \in \Gamma_{\ell} \subset \mathrm{GL}_{2 g}\left(\mathbb{Z}_{\ell}\right)$ and $n_{v} \in T_{\ell}(A) \cong \mathbb{Z}_{\ell}^{2 g}$. Since $\gamma_{v}$ does not have the eigenvalue 1 , we can invert the matrix $\gamma_{v}-$ id over $\mathbb{Q}_{\ell}$ and thus define

$$
m_{v}:=\left(\gamma_{v}-\mathrm{id}\right)^{-1} n_{v} \in V_{\ell}(A) \cong \mathbb{Q}_{\ell}^{2 g} \text {. }
$$


Let $\pi_{\ell}$ denote the natural composite homomorphism

$$
V_{\ell}(A) \rightarrow V_{\ell}(A) / T_{\ell}(A) \cong A\left[\ell^{\infty}\right] .
$$

Proposition 3.2. We have $\kappa_{v}(a)=\pi_{\ell}\left(m_{v}\right)$. In particular the order of the $\ell$-part of the reduction $a_{v}$ is equal to the $\ell$-part of the denominator of $m_{v}$.

Proof. The splitting $\lambda$ induces a decomposition

$$
V_{\ell}(A, a)=V_{\ell}(A) \oplus \mathbb{Q}_{\ell}
$$

which, as usual, we write in terms of column vectors. A direct calculation then shows that the eigenspace of $\tilde{\gamma}_{v}$ on $V_{\ell}(A, a)$ for the eigenvalue 1 is generated by the vector

$$
\left(\begin{array}{c}
-m_{v} \\
1
\end{array}\right)
$$

Thus again with respect to the decomposition induced by $\lambda$ the map

$$
\begin{aligned}
\mathbb{Z}[1 / \ell] & \longrightarrow \ell^{-\infty}(\mathbb{Z} a)=A\left[\ell^{\infty}\right] \oplus \mathbb{Z}[1 / \ell], \\
x & \mapsto\left(\begin{array}{c}
-\pi_{\ell}\left(x m_{v}\right) \\
x
\end{array}\right)
\end{aligned}
$$

defines a $\tilde{\gamma}_{v}$-equivariant splitting of 2.1 . The corresponding $\tilde{\gamma}_{v}$-equivariant splitting in the other direction

$$
A\left[\ell^{\infty}\right] \oplus \mathbb{Z}[1 / \ell]=\ell^{-\infty}(\mathbb{Z} a) \longrightarrow A\left[\ell^{\infty}\right]
$$

is given by

$$
\left(\begin{array}{l}
b \\
x
\end{array}\right)=\left(\begin{array}{c}
b+\pi_{\ell}\left(x m_{v}\right) \\
0
\end{array}\right)+\left(\begin{array}{c}
-\pi_{\ell}\left(x m_{v}\right) \\
x
\end{array}\right) \mapsto b+\pi_{\ell}\left(x m_{v}\right) .
$$

By Proposition 3.1 this map represents $\kappa_{v}$. Now since $\lambda$ is a special splitting, the element $a=\lambda(1)$ corresponds to the vector

$$
\left(\begin{array}{l}
0 \\
1
\end{array}\right) \text {. }
$$

It follows that $\kappa_{v}(a)=\pi_{\ell}\left(m_{v}\right)$, as desired.

\section{Density results for the $\ell$-part of the reduction}

In this section we derive several statements on the Dirichlet density of the set of places $v$ at which the $\ell$-part of the reduction of $a$ has certain properties. For all these statements we can disregard the finite set $S$ of places dividing $\ell$ or where $A$ has bad reduction. 
Theorem 4.1. Let $A$ be an abelian variety over a number field $K$ and $a \in A(K)$ a rational point of infinite order such that $\mathbb{Z} a$ is Zariski dense in A. Consider a rational prime and a point $b \in A\left[\ell^{\infty}\right]$. Then for all finite places $v$ of $K$ in a set of Dirichlet density $>0$ the $\ell$-part of the reduction of $a$ is equal to the reduction of $b$.

Proof. Choose a special splitting of 2.1 and let $U$ denote the set of elements

$$
\tilde{\gamma}=\left(\begin{array}{ll}
\gamma & n \\
0 & 1
\end{array}\right) \in \tilde{\Gamma}_{\ell} \subset\left(\begin{array}{cc}
\operatorname{Aut}_{\mathbb{Z}_{\ell}}\left(T_{\ell}(A)\right) & T_{\ell}(A) \\
0 & 1
\end{array}\right)
$$

satisfying $\operatorname{det}(\gamma-$ id $) \neq 0$. Clearly this is an open subset of $\tilde{\Gamma}_{\ell}$. Next $\tilde{\gamma} \mapsto$ $\pi_{\ell}\left((\gamma-\mathrm{id})^{-1} n\right)$ defines a continuous function from $U$ to the discrete set $A\left[\ell^{\infty}\right]$. It is therefore locally constant; hence

$$
U_{b}:=\left\{\begin{array}{l|l}
\tilde{\gamma} \in \tilde{\Gamma}_{\ell} & \begin{array}{l}
\operatorname{det}(\gamma-\mathrm{id}) \neq 0, \text { and } \\
\pi_{\ell}\left((\gamma-\mathrm{id})^{-1} n\right)=b
\end{array}
\end{array}\right\}
$$

is an open subset of $\tilde{\Gamma}_{\ell}$.

Lemma 4.2. $U_{b}$ is non-empty.

Proof. It suffices to show that the map

$$
U \rightarrow V_{\ell}(A),\left(\begin{array}{ll}
\gamma & n \\
0 & 1
\end{array}\right) \mapsto(\gamma-\mathrm{id})^{-1} n
$$

is surjective. This statement is invariant under conjugation by $V_{\ell}(A)$, and it suffices to prove it after replacing $K$ by a finite extension. Thus using Proposition 2.6 we may without loss of generality assume that

$$
\tilde{\Gamma}_{\ell}=\left(\begin{array}{cc}
\Gamma_{\ell} & N_{\ell} \\
0 & 1
\end{array}\right) .
$$

The desired statement is then equivalent to

$$
V_{\ell}(A)=\bigcup_{\substack{\gamma \in \Gamma_{\ell} \\ \operatorname{det}(\gamma-\mathrm{id}) \neq 0}}(\gamma-\mathrm{id})^{-1} N_{\ell} .
$$

Now $N_{\ell}$ is open in $T_{\ell}(A)$ by Corollary 2.9; hence $\ell^{r} T_{\ell}(A) \subset N_{\ell}$ for some integer $r$. On the other hand, for every integer $s>0$ there exists $\gamma \in \Gamma_{\ell}$ with $\operatorname{det}(\gamma-\mathrm{id}) \neq 0$ such that $\gamma \equiv \operatorname{id} \bmod \ell^{s}$. Indeed, any power $\rho_{\ell}\left(\text { Frob }_{v}\right)^{m}$ for a place $v \notin S$ and $m$ sufficiently divisible has these properties. For this element $\gamma$ we then have

$$
(\gamma-\mathrm{id}) T_{\ell}(A) \subset \ell^{s} T_{\ell}(A) \subset \ell^{s-r} N_{\ell}
$$

and hence

$$
\ell^{r-s} T_{\ell}(A) \subset(\gamma-\mathrm{id})^{-1} N_{\ell} .
$$

With $s \rightarrow \infty$ the desired equality follows. 
Now take any element $\tilde{\gamma} \in U_{b}$. By openness there exists an open normal subgroup $\tilde{\Delta} \triangleleft \tilde{\Gamma}_{\ell}$ such that $\tilde{\gamma} \tilde{\Delta} \subset U_{b}$. As $\tilde{\Gamma}_{\ell} / \tilde{\Delta}$ is the Galois group of a finite extension of $K$, by the Cebotarev density theorem there exists a set of places $v \notin S$ of $K$ of Dirichlet density $>0$ for which

$$
\tilde{\rho}_{\ell}\left(\operatorname{Frob}_{v}\right) \equiv \tilde{\gamma} \bmod \tilde{\Delta} .
$$

But for all these $v$ we have $\tilde{\rho}_{\ell}\left(\operatorname{Frob}_{v}\right) \in U_{b}$, which by Proposition 3.2 implies $\kappa_{v}(a)=b$. By the definition of $\kappa_{v}$ this means that the $\ell$-part of the reduction of $a$ is equal to the reduction of $b$, as desired.

Corollary 4.3. Let $A$ be an abelian variety over a number field $K$ and $a \in A(K)$ a rational point of infinite order such that $\mathbb{Z} a$ is Zariski dense in $A$. Consider a rational prime and an integer $r \geq 0$. Then for all finite places $v$ of $K$ in a set of Dirichlet density $>0$ the $\ell$-part of the reduction of a has order $\ell^{r}$.

Proof. Apply Theorem 4.1 to any point $b \in A\left[\ell^{\infty}\right]$ of order $\ell^{r}$. (This was also partly proved by Khare and Prasad [10, §5, Lemma 4-5].

Theorem 4.4. For $1 \leq i \leq d$ let $A_{i}$ be an abelian variety over a number field $K$ and $a_{i} \in A_{i}(K)$ a rational point of infinite order. Let $\ell$ be a rational prime. Then for all finite places $v$ of $K$ in a set of Dirichlet density $>0$ the $\ell$-part of the reduction of $a_{i}$ is non-trivial for every $i$.

Proof. We apply the results of the preceding sections to $A:=A_{1} \times \ldots \times A_{d}$ and $a:=\left(a_{1}, \ldots, a_{d}\right)$. Let $\mathrm{pr}_{i}: A \rightarrow A_{i}$ denote the projection to the $i^{\text {th }}$ factor. Then as in the proof of Theorem 4.1

$$
U^{\prime}:=\left\{\begin{array}{l|l}
\tilde{\gamma} \in \tilde{\Gamma}_{\ell} & \begin{array}{l}
\operatorname{det}(\gamma-\mathrm{id}) \neq 0, \text { and } \\
\forall i: \operatorname{pr}_{i} \pi_{\ell}\left((\gamma-\mathrm{id})^{-1} n\right) \neq 0
\end{array}
\end{array}\right\}
$$

is an open subset of $\tilde{\Gamma}_{\ell}$, and it suffices to prove:

\section{Lemma 4.5. $U^{\prime}$ is non-empty.}

Proof. We may replace $K$ by a finite extension. Thus using Proposition 2.6 we may without loss of generality assume that there exists $m \in V_{\ell}(A)$ such that

$$
\tilde{\Gamma}_{\ell}=\left(\begin{array}{ll}
1 & m \\
0 & 1
\end{array}\right) \cdot\left(\begin{array}{cc}
\Gamma_{\ell} & N_{\ell} \\
0 & 1
\end{array}\right) \cdot\left(\begin{array}{cc}
1 & -m \\
0 & 1
\end{array}\right)=\left\{\left(\begin{array}{cc}
\gamma & n-(\gamma-\mathrm{id}) m \\
0 & 1
\end{array}\right) \mid \begin{array}{l}
\gamma \in \Gamma_{\ell} \\
n \in N_{\ell}
\end{array}\right\} .
$$

We must therefore find $\gamma \in \Gamma_{\ell}$ and $n \in N_{\ell}$ such that $\operatorname{det}(\gamma-$ id $) \neq 0$ and

$$
\operatorname{pr}_{i} \pi_{\ell}\left((\gamma-\mathrm{id})^{-1} n-m\right)=\operatorname{pr}_{i} \pi_{\ell}\left((\gamma-\mathrm{id})^{-1}(n-(\gamma-\mathrm{id}) m)\right) \neq 0
$$

for all $i$. This second condition is equivalent to

$$
\operatorname{pr}_{i}\left((\gamma-\mathrm{id})^{-1} n\right) \not \equiv \operatorname{pr}_{i}(m) \bmod T_{\ell}\left(A_{i}\right) .
$$


Take any integer $r$ so that $\ell^{r} m \in T_{\ell}(A)$. Then it suffices to have

$$
\ell^{r} \operatorname{pr}_{i}\left((\gamma-\mathrm{id})^{-1} n\right) \notin T_{\ell}\left(A_{i}\right) .
$$

With $n=\left(n_{1}, \ldots, n_{d}\right) \in N_{\ell}$ this is equivalent to

$$
\ell^{r} n_{i} \notin(\gamma-\mathrm{id}) T_{\ell}\left(A_{i}\right) .
$$

Now by functoriality the image $\operatorname{pr}_{i}\left(N_{\ell}\right) \subset T_{\ell}\left(A_{i}\right)$ is the unipotent part of the $\ell$-adic Galois group attached to $\left(A_{i}, a_{i}\right)$. As $a_{i}$ has infinite order, this image is non-trivial by Theorem 2.8. Since any finite number of non-trivial linear inequalities in a free $\mathbb{Z}_{\ell}$-module can be simultaneously satisfied, we may therefore select $n=\left(n_{1}, \ldots, n_{r}\right) \in N_{\ell}$ such that all $n_{i} \neq 0$. Then clearly 4.6 holds for any suitable $\gamma \in \Gamma_{\ell}$ that is sufficiently close to the identity. This proves that $U^{\prime}$ is non-empty, as desired.

Theorem 4.7. For $1 \leq i \leq d$ let $A_{i}$ be an abelian variety over a number field $K$ and $a_{i} \in A_{i}(K)$ a rational point of infinite order. Then there exists a set of rational primes $\ell$ of Dirichlet density $>0$ with the following property. Let $f(T) \in \mathbb{Z}[T]$ be any polynomial which is a product of cyclotomic polynomials and a power of $T$. For any finite place $v$ of $K$ let $p_{v}$ denote the characteristic of the residue field and $a_{i, v}$ the reduction of $a_{i}$. Then for all finite places $v$ of $K$ in a set of Dirichlet density $>0$ the $\ell$-part of $f\left(p_{v}\right) a_{i, v}$ is non-trivial for every $i$.

Proof. We apply the results of the preceding sections to $A:=A_{1} \times \ldots \times A_{d}$ and $a:=\left(a_{1}, \ldots, a_{d}\right)$. By Corollary 1.3 there exists a set of rational primes $\ell$ of positive Dirichlet density for which the associated algebraic monodromy group $G_{\ell}$ splits over $\mathbb{Q}_{\ell}$. We will prove the theorem for any such $\ell$.

Let $\mu: G_{\ell} \rightarrow \mathbb{G}_{m, \mathbb{Q}_{\ell}}$ be the multiplier character and let $\mathrm{pr}_{i}: A \rightarrow A_{i}$ denote the projection to the $i^{\text {th }}$ factor. As in the proof of Theorem 4.1

$$
U_{f}:=\left\{\begin{array}{l|l}
\tilde{\gamma} \in \tilde{\Gamma}_{\ell} & \begin{array}{l}
\operatorname{det}(\gamma-\mathrm{id}) \neq 0, \text { and } \\
\forall i: f(\mu(\gamma)) \operatorname{pr}_{i} \pi_{\ell}\left((\gamma-\mathrm{id})^{-1} n\right) \neq 0
\end{array}
\end{array}\right\}
$$

is an open subset of $\tilde{\Gamma}_{\ell}$.

Lemma 4.8. $U_{f}$ is non-empty.

Proof. As in the proof of Lemma 4.5, after replacing $K$ by a finite extension we may assume that

$$
\tilde{\Gamma}_{\ell}=\left\{\begin{array}{l|l}
\left(\begin{array}{cc}
\gamma & n-(\gamma-\mathrm{id}) m \\
0 & 1
\end{array}\right) \mid \begin{array}{l}
\gamma \in \Gamma_{\ell} \\
n \in N_{\ell}
\end{array}
\end{array}\right\}
$$

for some $m \in V_{\ell}(A)$. We must therefore find elements $\gamma \in \Gamma_{\ell}$ and $n \in N_{\ell}$ such that $\operatorname{det}(\gamma-\mathrm{id}) \neq 0$ and

$$
f(\mu(\gamma)) \operatorname{pr}_{i} \pi_{\ell}\left((\gamma-\mathrm{id})^{-1} n-m\right) \neq 0
$$


for every $i$. This second condition is equivalent to

$$
f(\mu(\gamma)) \operatorname{pr}_{i}\left((\gamma-\mathrm{id})^{-1} n\right) \not \equiv f(\mu(\gamma)) \operatorname{pr}_{i}(m) \bmod T_{\ell}\left(A_{i}\right) .
$$

Taking any integer $r$ so that $\ell^{r} m \in T_{\ell}(A)$, it suffices to have

$$
\ell^{r} f(\mu(\gamma)) \operatorname{pr}_{i}\left((\gamma-\mathrm{id})^{-1} n\right) \notin T_{\ell}\left(A_{i}\right) .
$$

Now by the assumption on $\ell$ there exists a split maximal torus $S_{\ell} \subset G_{\ell}$. Every character $\chi$ of $S_{\ell}$ is then defined over $\mathbb{Q}_{\ell}$. For any representation $W$ of $S_{\ell}$ let $\mathrm{pr}_{\chi}: W \rightarrow W_{\chi}$ denote the projection to the weight space associated to $\chi$. Recall from Proposition 2.5 that $N_{\ell}$ is open in $U_{\ell}$, which is an algebraic representation of $G_{\ell}$ and hence of $S_{\ell}$. Thus $N_{\ell, \chi}:=V_{\ell}(A)_{\chi} \cap N_{\ell}$ is open in the weight space $U_{\ell, \chi}$. For every $\chi$ we want to select an element $n_{\chi} \in N_{\ell, \chi}$ such that for all $i$ we have $\operatorname{pr}_{i}\left(n_{\chi}\right) \neq 0$ whenever $\operatorname{pr}_{i}\left(U_{\ell, \chi}\right) \neq 0$. This is possible, because any finite number of non-trivial linear inequalities in a free $\mathbb{Z}_{\ell}$-module can be simultaneously satisfied. We will show the desired assertions with $n:=\sum_{\chi} n_{\chi} \in N_{\ell}$ and a suitable element $\gamma \in S_{\ell}\left(\mathbb{Q}_{\ell}\right) \cap \Gamma_{\ell}$. To satisfy 4.9 it suffices to have

$$
\forall i \exists \chi: \ell^{r} f(\mu(\gamma)) \operatorname{pr}_{i}\left((\gamma-\mathrm{id})^{-1} n_{\chi}\right) \notin \operatorname{pr}_{\chi}\left(T_{\ell}\left(A_{i}\right)\right) .
$$

As $n_{\chi}$ is an eigenvector of $\gamma$ for the eigenvalue $\chi(\gamma) \in \mathbb{Z}_{\ell}$, this element is equal to

$$
\frac{\ell^{r} f(\mu(\gamma))}{\chi(\gamma)-1} \cdot \operatorname{pr}_{i}\left(n_{\chi}\right) .
$$

Fix an integer $s$ so that for all $i$ and $\chi$ with $\operatorname{pr}_{i}\left(n_{\chi}\right) \neq 0$ we have

$$
\operatorname{pr}_{i}\left(n_{\chi}\right) \notin \ell^{s} \operatorname{pr}_{\chi}\left(T_{\ell}\left(A_{i}\right)\right) \text {. }
$$

By construction this affects all pairs $(i, \chi)$ with $\operatorname{pr}_{i}\left(U_{\ell, \chi}\right) \neq 0$. Thus it suffices to prove the following assertion, from which the $n_{\chi}$ have vanished.

Sublemma 4.10. There exists an element $\gamma \in S_{\ell}\left(\mathbb{Q}_{\ell}\right) \cap \Gamma_{\ell} \operatorname{satisfying} \operatorname{det}(\gamma-\mathrm{id}) \neq$ 0 such that for every $i$ there exists a character $\chi$ with $\operatorname{pr}_{i}\left(U_{\ell, \chi}\right) \neq 0$ and

$$
\operatorname{ord}_{\ell}(\chi(\gamma)-1) \geq r+s+\operatorname{ord}_{\ell}(f(\mu(\gamma))) \text {. }
$$

Proof. For every $i$ let $B_{i} \subset A_{i}$ be the identity component of the Zariski closure of $\mathbb{Z} a_{i}$. Applying Proposition 1.5 to $B:=B_{1} \times \ldots \times B_{d}$ shows that there exist weights $\chi_{i}$ of $S_{\ell}$ on $V_{\ell}\left(B_{i}\right) \subset V_{\ell}\left(A_{i}\right)$ so that $\mu$ is $\mathbb{Q}$-linearly independent of $\chi_{1}, \ldots, \chi_{d}$. The functoriality and Theorem 2.8 together imply that $\operatorname{pr}_{i}\left(U_{\ell}\right)=V_{\ell}\left(B_{i}\right)$. Since the projection map $\mathrm{pr}_{i}$ is $S_{\ell}$-equivariant, we deduce that

$$
\operatorname{pr}_{i}\left(U_{\ell, \chi_{i}}\right)=V_{\ell}\left(B_{i}\right)_{\chi_{i}} \neq 0 \text {. }
$$

It remains to find an element $\gamma \in S_{\ell}\left(\mathbb{Q}_{\ell}\right) \cap \Gamma_{\ell}$ with $\operatorname{det}(\gamma-\mathrm{id}) \neq 0$ and for all $i$

$$
\operatorname{ord}_{\ell}\left(\chi_{i}(\gamma)-1\right) \geq r+s+\operatorname{ord}_{\ell}(f(\mu(\gamma))) .
$$


The inequality 4.11 means that $\chi_{i}(\gamma)$ is much closer to the identity than $\mu(\gamma)$. To be precise let us first shrink $\Gamma_{\ell}$ so that $\Gamma_{\ell}$ acts trivially on $T_{\ell}(A) / \ell^{2} T_{\ell}(A)$. Then for every element $\gamma \in S_{\ell}\left(\mathbb{Q}_{\ell}\right) \cap \Gamma_{\ell}$ we have $\mu(\gamma) \equiv 1 \bmod \ell^{2}$. On the other hand choose an integer $k>0$ such that all non-zero roots of $f(T)$ are roots of unity of order dividing $k$ and have multiplicity $\leq k$. Then after multiplying $f(T)$ by some more cyclotomic polynomials we may assume that $f(T)=T^{k^{\prime}}\left(T^{k}-1\right)^{k}$ for some $k^{\prime} \geq 0$. A standard calculation now shows that

$$
\begin{aligned}
\operatorname{ord}_{\ell}(f(\mu(\gamma))) & =k^{\prime} \cdot \operatorname{ord}_{\ell}(\mu(\gamma))+k \cdot \operatorname{ord}_{\ell}\left(\mu(\gamma)^{k}-1\right) \\
& =k \cdot \operatorname{ord}_{\ell}(k)+k \cdot \operatorname{ord}_{\ell}(\mu(\gamma)-1) .
\end{aligned}
$$

Setting $t:=r+s+k \cdot \operatorname{ord}_{\ell}(k)$ we thus need to find an element $\gamma \in S_{\ell}\left(\mathbb{Q}_{\ell}\right) \cap \Gamma_{\ell}$ with $\operatorname{det}(\gamma-\mathrm{id}) \neq 0$ and for all $i$

$$
\operatorname{ord}_{\ell}\left(\chi_{i}(\gamma)-1\right) \geq t+k \cdot \operatorname{ord}_{\ell}(\mu(\gamma)-1) .
$$

To achieve this let $S_{\ell}^{1}$ denote the identity component of $\operatorname{Ker}\left(\mu \mid S_{\ell}\right)$, which is a subtorus of codimension 1 . Since $\mu$ is $\mathbb{Q}$-linearly independent of $\chi_{1}, \ldots, \chi_{d}$ and $S_{\ell}$ splits over $\mathbb{Q}_{\ell}$, there exists a subtorus $S_{\ell}^{2}$ of dimension 1 inside $\bigcap_{i=1}^{d} \operatorname{Ker}\left(\chi_{i} \mid S_{\ell}\right)$ on which $\mu$ is non-trivial. We will take $\gamma=\gamma_{1} \gamma_{2}$ with $\gamma_{1} \in S_{\ell}^{1}\left(\mathbb{Q}_{\ell}\right) \cap \Gamma_{\ell}$ and $\gamma_{2} \in S_{\ell}^{2}\left(\mathbb{Q}_{\ell}\right) \cap \Gamma_{\ell}$. Then the left hand side of 4.12 depends only on $\gamma_{1}$, while the right hand side depends only on $\gamma_{2}$.

Theorem 1.1 (c) implies that $\Gamma_{\ell}$ contains an open subgroup of $S_{\ell}\left(\mathbb{Q}_{\ell}\right)$. Thus if we first select any non-trivial $\gamma_{2}$, the inequality 4.12 will hold for every $\gamma_{1}$ that is sufficiently close to the identity. Furthermore, none of the weights of $S_{\ell}$ on $V_{\ell}(A)$ is zero, e.g., by Proposition 1.4. Thus in any neighborhood of the identity $\gamma_{1}$ can be chosen such that $\gamma=\gamma_{1} \gamma_{2}$ does not have the eigenvalue 1 on $V_{\ell}(A)$, which means that $\operatorname{det}(\gamma-\mathrm{id}) \neq 0$. Thus all requirements can be simultaneously satisfied, finishing the proof of Sublemma 4.10 and hence of Lemma 4.8.

Now we return to the proof of Theorem 4.7. Since $U_{f} \subset \tilde{\Gamma}_{\ell}$ is a non-empty open subset, as in the proof of Theorem 4.1 we conclude that there exists a set of places $v \notin S$ of $K$ of Dirichlet density $>0$ for which $\tilde{\rho}_{\ell}\left(\right.$ Frob $\left._{v}\right) \in U_{f}$. We may also assume that the associated residue fields $k_{v}$ have prime order, because the remaining places form a set of Dirichlet density 0 . For these places we have $\mu \rho_{\ell}\left(\operatorname{Frob}_{v}\right)=\left|k_{v}\right|=p_{v}$. The definition of $U_{f}$ and Proposition 3.2 thus imply that $f\left(p_{v}\right) \operatorname{pr}_{i} \kappa_{v}(a) \neq 0$ for every $i$. By the definition of $\kappa_{v}$ this means that the $\ell$-part of $f\left(p_{v}\right) a_{i, v}$ is non-trivial for every $i$, as desired.

Remark 4.13. Theorem 4.7 is not true in general for every rational prime $\ell$, even for a single abelian variety $A$ and a single rational point $a \in A(K)$. For a counterexample suppose that $A$ is an elliptic curve with complex multiplication over $K$. Then $\operatorname{End}_{K}(A)$ is an order in an imaginary quadratic number field $F$, and for any rational prime $\ell$ the image of Galois is an open compact subgroup of $\left(F \otimes \mathbb{Q}_{\ell}\right)^{*}$. 
Thus $G_{\ell}$ splits over $\mathbb{Q}_{\ell}$ if and only if $\ell$ splits in $F$, and in this case the proof of Theorem 4.7 goes through.

If $\ell$ does not split in $F$, we will show that the theorem is false. It is known that for every finite place $v \notin S$ with $\left|k_{v}\right|=p_{v}$ the element $\alpha_{v}:=\rho_{\ell}\left(\right.$ Frob $\left._{v}\right)$ is an algebraic integer in $F$ with $\alpha_{v} \bar{\alpha}_{v}=p_{v}$ and that the cardinality of $A_{v}\left(k_{v}\right)$ is equal to $\left(\alpha_{v}-1\right)\left(\bar{\alpha}_{v}-1\right)$. In particular the integer $\left(\alpha_{v}-1\right)\left(\bar{\alpha}_{v}-1\right)$ annihilates the reduction of $a$. Now the fact that $F$ has only one prime above $\ell$ implies that

$$
\operatorname{ord}_{\ell}\left(\alpha_{v}-1\right)=\operatorname{ord}_{\ell}\left(\bar{\alpha}_{v}-1\right) \leq \operatorname{ord}_{\ell}\left(\alpha_{v} \bar{\alpha}_{v}-1\right)=\operatorname{ord}_{\ell}\left(p_{v}-1\right) .
$$

Thus with $f(T):=(T-1)^{2}$ we deduce that

$$
\operatorname{ord}_{\ell}\left(\left(\alpha_{v}-1\right)\left(\bar{\alpha}_{v}-1\right)\right) \leq 2 \cdot \operatorname{ord}_{\ell}\left(p_{v}-1\right)=\operatorname{ord}_{\ell}\left(f\left(p_{v}\right)\right) .
$$

This implies that $f\left(p_{v}\right)$ annihilates the $\ell$-part of the reduction of $a$. Since this is so for every $v \notin S$, we conclude that in this example Theorem 4.7 is true precisely for $\ell$ in a set of Dirichlet density $1 / 2$.

\section{Density results for the full reduction}

In this section we derive some consequences of the density results of the preceding section which no longer refer to any particular prime $\ell$.

Theorem 5.1. For $1 \leq i \leq d$ let $A_{i}$ be an abelian variety over a number field $K$ and $a_{i} \in A_{i}(K)$ a rational point. Assume that for all finite places $v$ of $K$ in a set of Dirichlet density 1 the reduction of at least one $a_{i}$ is annihilated by a power of the residue characteristic $p_{v}$. Then at least one $a_{i}=0$.

Proof. Suppose that some $a_{i}$ is a torsion point of order $n$. If $n=1$, we are done. Otherwise the order of the reduction of $a_{i}$ at any finite place $v \nmid n$ is still $n$, and therefore not a power of $p_{v}$. Thus after removing $A_{i}$ and $a_{i}$ from the list the assumptions still hold. After iterating this we may assume that all $a_{i}$ have infinite order; we must then derive a contradiction. Select any rational prime $\ell$. Then by Theorem 4.4 for all finite places $v \nmid \ell$ of $K$ in a set of Dirichlet density $>0$ the $\ell$-part of the reduction of every $a_{i}$ is non-trivial. In particular, these reductions are not annihilated by a power of $p_{v}$, contradicting the given assumption.

Remark 5.2. Damian Roessler pointed out to the author that Theorem 5.1 can also be deduced from a theorem of Wong [16]. To sketch this set $A:=A_{1} \times \ldots \times A_{d}$. For any prime $\ell$ let $\Gamma_{\ell, 1}$ denote the image of $\operatorname{Gal}(\bar{K} / K)$ in its action on the $\ell$ torsion subgroup $A[\ell]$. By a theorem of Serre, which for example follows from [15, Th. 2], the group cohomology $H^{1}\left(\Gamma_{\ell, 1}, A[\ell]\right)$ vanishes for all $\ell \gg 0$. We temporarily fix any such $\ell>d$.

The assumptions in Theorem 5.1 imply that for all $v$ in a set of Dirichlet density 1 the reduction of at least one $a_{i}$ has trivial $\ell$-part. Since multiplication by 
$\ell$ induces an automorphism on the prime-to- $\ell$ part of $A_{v}\left(k_{v}\right)$, the reduction of $a_{i}$ then lies in $\ell A_{v}\left(k_{v}\right)$. Wong [16, Th. 2] deduces from this that at least one $a_{i}$ is contained in $\ell A(K)$. Since this is true for every $\ell \gg 0$, and the Mordell-Weil group $A(K)$ is finitely generated, this implies that at least one $a_{i}$ is torsion. As in the proof of 5.1 we now deduce that at least one $a_{i}=0$, as desired.

Theorem 5.3. For $1 \leq i \leq d$ let $A_{i}$ be an abelian variety over a number field $K$ and $a_{i} \in A_{i}(K)$ a rational point. Let $f(T) \in \mathbb{Z}[T]$ be any polynomial which is a product of cyclotomic polynomials and a power of $T$. For any finite place $v$ of $K$ let $p_{v}$ denote the characteristic of the residue field and $a_{i, v}$ the reduction of $a_{i}$. Assume that for all finite places $v$ of $K$ in a set of Dirichlet density 1 at least one $a_{i, v}$ is annihilated by $f\left(p_{v}\right)$. Then at least one $a_{i}$ is a torsion point.

Proof. Suppose that every $a_{i}$ has infinite order. Then by Theorem 4.7 there exists a rational prime $\ell$ such that for all finite places $v$ of $K$ in a set of Dirichlet density $>0$ the $\ell$-part of every $f\left(p_{v}\right) a_{i, v}$ is non-trivial. In particular, these $a_{i, v}$ are not annihilated by $f\left(p_{v}\right)$, contradicting the given assumption. Thus the order of at least one $a_{i}$ is finite.

\section{References}

[1] Bashmakov, M.: The cohomology of abelian varieties over a number field. Russian Math. Surveys 27(6), 25-70 (1977)

[2] Bertrand, D.: Galois representations and transcendental numbers. New advances in transcendence theory (Durham, 1986), Cambridge: Cambridge Univ. Press 1988, pp. 37-55

[3] Bogomolov, F.A.: Points of finite order on abelian varieties. (Russian) Izv. Akad. Nauk SSSR Ser. Mat. 44, 782-804 (1980) 973 = Math. USSR Izvestija 17, 55-72 (1981)

[4] Bogomolov, F.A.: Sur l'algébricité des représentations $\ell$-adiques. C. R. Acad. Sci. Paris Sér. A-B 290(15), A701-A703 (1980)

[5] Borel, A.: Linear Algebraic Groups. GTM 126, New York etc.: Springer 1991

[6] Corrales-Rodrigáñez, C., Schoof, R.: The Support Problem and Its Elliptic Analogue. J. Number Th. 64, 276-290 (1997)

[7] Deligne, P.: Théorie de Hodge, III. Publ. Math. IHES 44, 5-77 (1974)

[8] Faltings, G.: Finiteness Theorems for Abelian Varieties over Number Fields. Arithmetic Geometry, G. Cornell, J.H. Silverman (Eds.), New York etc.: Springer 1986, pp. 9-27.

[9] Hindry, M.: Autour d'une conjecture de Serge Lang. Invent. math. 94, 575-603 (1988)

[10] Khare, C., Prasad, D.: Reduction of Homomorphisms mod $p$ and algebraicity. Preprint (18 p.) arXiv:math.NT/0211004 v1 1 Nov 2002

[11] Larsen, M.J.: The Support Problem For Abelian Varieties. Preprint (7 p.) arXiv: math. NT/0211118 v3 28 Feb 2003

[12] Pink, R., Roessler, D.: A Conjecture of Beauville and Catanese Revisited. Math. Ann. (2004) DOI: 10.1007/s00208-004-0549-7

[13] Ribet, K.: Kummer theory on extensions of abelian varieties by tori. Duke Math. J. 46(4), 745-761 (1979)

[14] Serre, J.-P.: Lettre à Ken Ribet du 1/1/1981. Oeuvres vol. IV Berlin etc.: Springer 2000, pp. $1-17$

[15] Serre, J.-P.: Résumé des cours de 1985-1986. Annuaire du Collège de France (1986), 95-99 = Oeuvres vol. IV, Berlin Heidelberg New York: Springer 2000, pp. 33-37

[16] Wong, S.: Power Residues on Abelian Varieties. Manuscripta math. 102, 129-137 (2000) 